\title{
DESEMPENHO DE UM REATOR SEQUENCIAL COM BIOFILME EM LEITO MÓVEL SOB DIFERENTES CONCENTRAÇÕES DE OXIGÊNIO
}

\section{PERFORMANCE OF A SEQUENTIAL MOVING BED BIOFILM REACTOR UNDER DIFFERENT DISSOLVED OXYGEN CONCENTRATIONS}

\section{Rodrigo de Freitas Bueno', Marianella Munoz Rivera², Carolina Leni' ${ }^{2}$}

\author{
${ }^{1}$ Universidade de São Paulo - Escola Politécnica - Dep. de Engenharia Hidráulica e \\ Sanitária (PHA). Av. Almeida Prado, 83 transv. 2, Cidade Universitária, CEP 05508- \\ 900, São Paulo, SP. E-mail: robueno@usp.br \\ ${ }^{2}$ University of Antioquia, Colômbia. E-mail: marianellamovie1@gmail.com; \\ clenis875@gmail.com
}

\begin{abstract}
RESUMO
O objetivo do estudo foi avaliar o comportamento de um sistema piloto contendo suportes plásticos móveis (carries) para tratamento de esgoto sanitário em diferentes concentrações de oxigênio dissolvido (OD). Para avaliação do processo foram colocados em operação dois reatores sob condições iguais, diferindo apenas pela introdução em um deles do meio suporte (denominado SMBBR; aquele sem meio suporte, de SBR). O estudo teve duas etapas principais, na primeira os sistemas foram operados com OD na faixa de 1,5-2,0 $\mathrm{mgO}_{2} / \mathrm{L}$ (valor usual para esse tipo de processo) que resultou em uma remoção de DQO superior a $90 \%$, de nitrogênio e fósforo total superior a 78\%, em ambos os reatores. Na Etapa II, os sistemas foram operados com uma concentração de OD na faixa de $0,3-0,8 \mathrm{mgO}_{2} / \mathrm{L}$, a fim de se avaliar o efeito da diminuição da concentração de OD na remoção de material orgânico e nutrientes. Os resultados nessa etapa mostraram uma remoção de DQO e de nitrogênio total superior a $90 \%$ e fósforo total de $83 \%$. Ao ser comparados os resultados entre as etapas, pode-se dizer que a diminuição da concentração de OD não afetou a remoção de matéria orgânica e nutrientes. Além do fato de melhorar a remoção de nitrogênio total potencializando a desnitrificação, o maior ganho nessa configuração operacional está relacionado ao gasto de energia elétrica requerido para a aeração do sistema, onde pode se chegar a uma redução de $68 \%$ a menos que os processos tradicionais. Ainda, durante a operação dos sistemas o processo de SMBBR se mostrou mais robusto, com estabilidade operacional frente a variações de carga orgânica afluente.

Palavras-chave: Lodo ativado. SMBBR. SBR. Nitrificação e Desnitrificação Simultânea. Remoção biológica de fósforo
\end{abstract}

ABSTRACT

The study evaluated the behavior of a mobile pilot containing plastic substrates system (carries) for treatment of domestic sewage in different Dissolved oxygen (DO). For evaluation of the process 
were put into operation two reactors under equal conditions, differing only by the introduction in one of the support means (called SMBBR; that without the support medium, SBR). The study had two main steps, the first systems were operated in the range of 1.5-2.0 $\mathrm{DO} \mathrm{mgO}_{2} / \mathrm{L}$ (typical value for such a procedure) resulted in a COD removal exceeding 90\%, nitrogen and total phosphorus exceeding $78 \%$ in both reactors. In Step II, the systems were operated with a DO concentration in the range of $0.3-0.8 \mathrm{mgO}_{2} / \mathrm{L}$, in order to evaluate the effect of lowering the $\mathrm{DO}$ concentration in the removal of organic material, and strengthening the process of denitrification. The results at this stage showed a COD removal and total nitrogen exceeding $90 \%$ and $83 \%$ total phosphorus. When comparing the results between steps, it can be said that the decrease in DO concentration did not affect the removal of organic matter and nutrients, and the fact improve the removal of total nitrogen the biggest gain this operating configuration is related to spending energy required for aeration system where you can get a reduction of $68 \%$ less than traditional processes. Further, during operation of the system SMBBR process was more stable than the SBR operable not is being adversely affected by the influent load variations.

Keywords: Activated sludge systems. SMBBR. SBR. Simultaneous nitrification and denitrification (SND). Biological excess phosphorus removal.

\section{INTRODUÇÃO}

O processo de tratamento conhecido por Moving Bed Biofilm Reactors - MBBR baseia-se no desenvolvimento de biofilme em leito móvel, para degradação de matéria orgânica e remoção de nutrientes do esgoto sanitário. Quando associado ao processo de lodo ativado cultiva-se, ao mesmo tempo, a biomassa aderida aos corpos plásticos e a biomassa em suspensão, o que confere ao sistema grande capacidade de carga em termos de matéria orgânica. Uma das possibilidades é seu uso hibrido em reatores de lodo ativado operado em bateladas sequenciais, processo conhecido como Sequential Moving Bed Biofilm Reactors - SMBBR e que constitui alternativa atraente para ampliação da capacidade nominal de uma estação de tratamento, ou quando se objetiva a remoção de nutrientes sem aumento de volume dos tanques (ANDREOTTOLA et al., 2000; KIM et al., 2011; ACCINELLI et al., 2012; FUJII et al., 2013).

Biofilmes consistem em comunidades de microrganismos desenvolvidas sobre superfícies. Estes elementos de suporte podem ser pequenas peças de polietileno que apresentam extensa área superficial específica e se encontram suspensas e em movimento no lodo do reator. A agitação é proporcionada pelo próprio sistema de aeração, constituído por difusores de ar instalados no fundo do tanque (HEM et al., 1994).

As substâncias consideradas poluentes, matéria orgânica, nitrogênio na forma amoniacal e fósforo, são os substratos para o crescimento da massa bacteriana cuja concentração, composta pela parcela aderida ao meio suporte e pela parcela de biomassa em suspensão, é muito maior do que no sistema convencional (apenas com biomassa suspensa) (FUJII, 2011).

A atividade microbiológica também é mais alta devido à grande variedade de populações microbiológicas nos biofilmes (RUSTEN et al., 1995), possibilitando maior remoção de substratos. Os biofilmes têm como característica importante à estabilidade a variações operacionais, sejam de carga, vazão, ou composição do esgoto afluente. Uma vez que as culturas estejam plenamente estabelecidas no meio suporte móvel oferecem robustez ao sistema, resistindo a condições que o lodo em suspensão não seria capaz (AYGUN et al., 2008).

A nitrificação é beneficiada por esse efeito de estabilidade, na medida em que as bactérias nitrificantes também se desenvolvem nessas comunidades que compõe o biofilme (ØDEGAARD et al., 1993). Nos sistemas de SMBBR e SBR em estudo, o processo de nitrificação e desnitrificação simultânea (NDS) se desenvolvem nos reatores, devido ao emprego de um sistema automatizado de 
controle da concentração de OD, de forma que o fornecimento de ar seja feito de acordo com a demanda por oxigênio, decorrente da carga orgânica afluente a cada momento (CHIU et al., 2007). Dessa forma, ao manter-se baixa a concentração de oxigênio dissolvido no licor misto (na faixa de 0,3 a $0,8 \mathrm{mgO}_{2} / \mathrm{L}$ ), possibilita-se o surgimento de microrregiões anóxicas no interior dos flocos.

Dependendo da concentração de OD, de amônia e de DQO biodegradável, o oxigênio dissolvido poderá ser consumido rapidamente no interior do floco, tornando-se indisponível na camada interna (ZHAO et al., 1999; ZHANG et al., 2007). O nitrato produzido na zona aeróbia pode difundir-se em direção à camada interna conjuntamente com o substrato, possibilitando a ocorrência da desnitrificação. A remoção biológica de nitrogênio nessas condições pode ser obtida com ótimo desempenho e estabilidade, conforme mostra o estudo realizado por Bueno (2011) operando sistema de lodo ativado com aeração prolongada sob baixa concentração de OD e obteve uma remoção superior a $90 \%$ de nitrogênio total.

A remoção do fósforo está ligada ao desenvolvimento de populações de organismos capazes de acumular grande quantidade deste elemento no interior de suas células (conhecidos como Organismos Acumuladores de Fósforo - OAP). O cultivo dos OAPs no sistema é realizado através da existência de uma etapa anaeróbia no processo, que garante um ambiente onde há uma vantagem competitiva para esses organismos: eles são capazes de utilizar uma fonte interna de energia (polifosfato previamente acumulado na etapa aeróbia) para sequestrar na etapa anaeróbia parte da matéria orgânica rapidamente biodegradável (WENTZEL et al., 1990; HELNESS et al. 2001; GIESEKE et al., 2002; ZENG et al., 2003). O presente trabalho, além de objetivar o estudo comparativo entre o processo de lodo ativado com e sem a presença de meio suporte, avaliou o desempenho desses sistemas em relação à remoção de material orgânico, nutrientes e o processo de NDS.

\section{MATERIAIS E MÉTODOS}

\subsection{Configuração e operação da planta piloto}

A pesquisa foi desenvolvida por meio de experimento realizado em uma estação de tratamento de esgotos (ETE), em escala piloto, no Centro Tecnológico de Hidráulica da Escola Politécnica da USP. Foram postos em marcha dois processos de lodo ativado de dimensões iguais (Reator 1 -SBR) e (Reator 2 - SMBBR), diferindo apenas pela introdução em um deles de suportes móveis para o desenvolvimento de biomassa aderida (SMBBR). A operação do sistema teve um período de 09 meses compreendendo o ano de 2014. O esgoto sanitário era proveniente do CRUSP - Conjunto Residencial da USP, recebendo tratamentos prévios de peneiramento, desarenação, remoção de gordura e decantação primária. O sistema piloto que representou o processo SMBBR recebeu anéis plásticos móveis do tipo Kaldnes ${ }^{\circledR}$ com área superficial específica estimada em 310 $\mathrm{m}^{2} / \mathrm{m}^{3}$ que ocuparam $50 \%$ do volume útil do reator. A Figura 1 mostra o arranjo experimental utilizado neste estudo. 


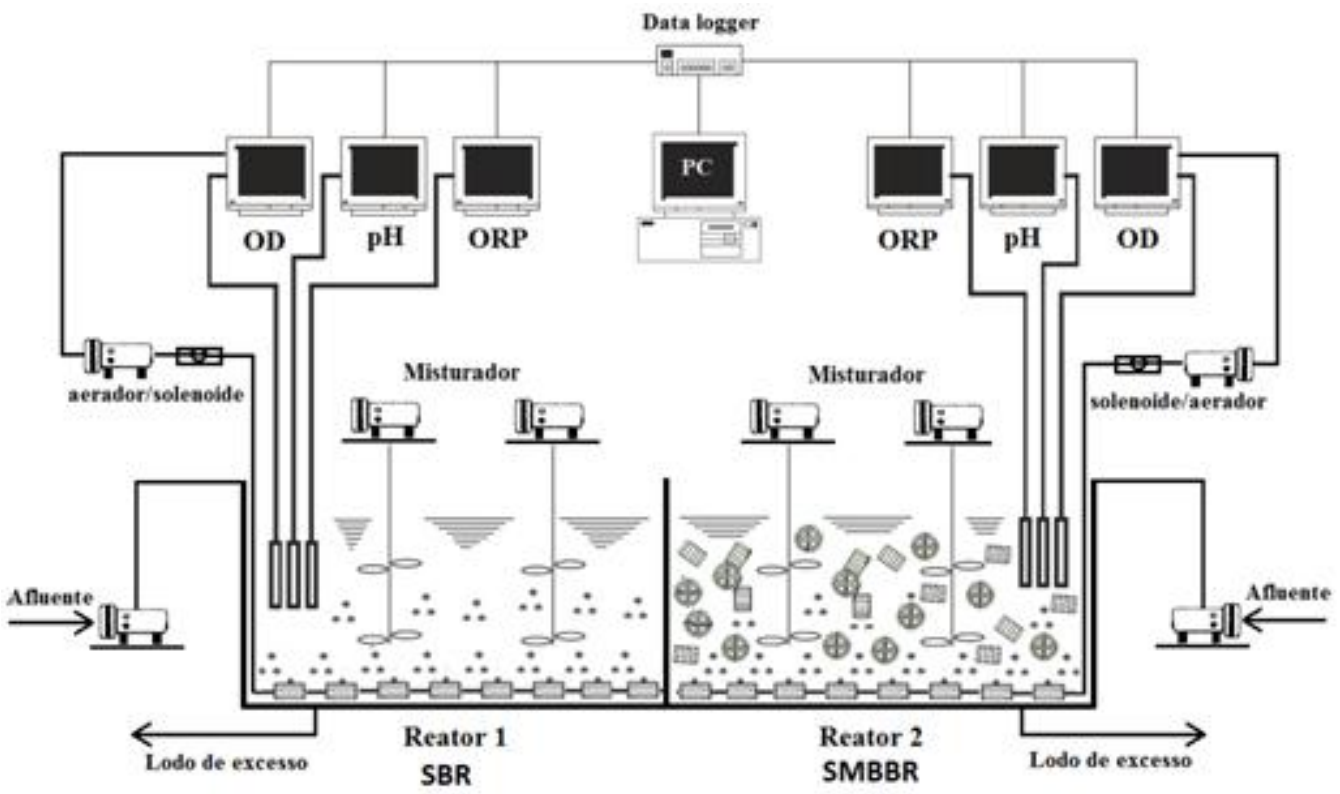

Figura 1. Corte esquemático do sistema de lodo ativado SBR e SMBBR.

Segundo o delineamento experimental proposto, os dois sistemas operaram simultânea e paralelamente. Foram realizadas duas etapas de operação, sendo a primeira sob concentração de OD de 1,5-2,0 $\mathrm{mgO}_{2} / \mathrm{L}$ para avaliação da nitrificação e desnitrificação em um ciclo convencional e a segunda de 0,3-0,8 $\mathrm{mgO}_{2} / \mathrm{L}$, com objetivo de se obter a nitrificação e desnitrificação simultânea (NDS) conforme estudos realizados por Zhao et al. (1999), Zhang, et al. (2007), Thauré et al. (2008) e Bueno (2011). A Tabela 1 mostra as condições operacionais utilizadas neste estudo. O tempo do ciclo foi baseado em Munch et al., (1996) e Guo et al., (2007) que operaram sistemas de SBR e SMBBR em condições similares ao do presente estudo. A Figura 2 mostra o ciclo operacional proposto para cada etapa.

Tabela 1 - Condições operacionais empregadas no reator SBR e SMBBR para se obter a nitrificação e desnitrificação simultânea.

\begin{tabular}{|c|c|c|c|c|c|}
\hline \multicolumn{2}{|c|}{ Condições Operacionais } & \multicolumn{2}{|c|}{ Etapa I } & \multicolumn{2}{|c|}{ Etapa II } \\
\hline Reator & & SBR & SMBBR & SBR & SMBBR \\
\hline Volume útil (L) & 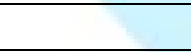 & 36 & 36 & 36 & 36 \\
\hline Dias de operaçã & 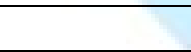 & 64 & 64 & 46 & 46 \\
\hline TRS (dias) & & 20 & 20 & 20 & 20 \\
\hline Troca volumétri & $\%)$ & 50 & 50 & 50 & 50 \\
\hline TDH (horas) & & 6 & 6 & 6 & $\bar{c}$ \\
\hline \multirow{2}{*}{$\operatorname{SSVLM}(\mathrm{mg} / \mathrm{L})$} & Suspensão & $1735 \pm 522$ & $1625 \pm 420$ & $1860 \pm 375$ & $1642 \pm 490$ \\
\hline & Aderida & - & $937 \pm 330$ & - & $1110 \pm 320$ \\
\hline $\mathrm{OD}\left(\mathrm{mgO}_{2} / \mathrm{L}\right)$ & & $1,5-2,0$ & $1,5-2,0$ & $0,3-0,8$ & $0,3-0,8$ \\
\hline
\end{tabular}


Ciclo Etapa I

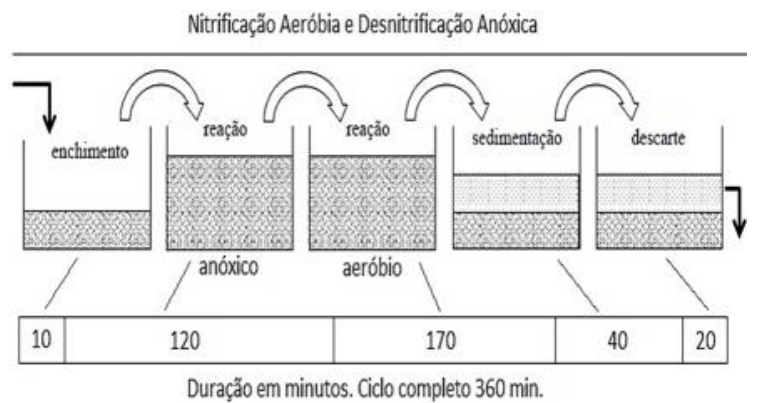

Ciclo Etapa II

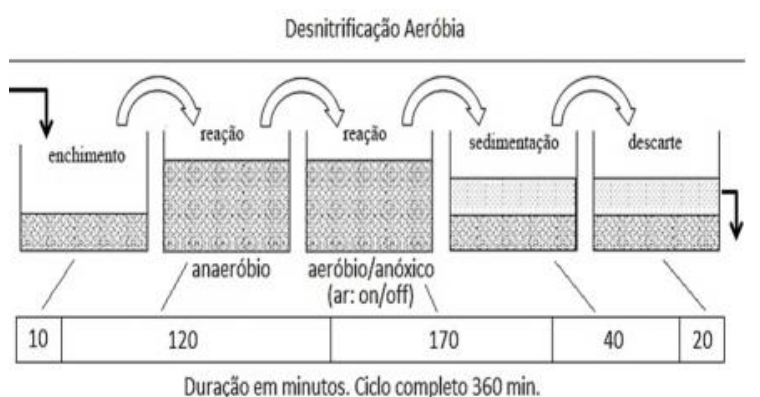

Figura 2. Condições operacionais utilizadas na Etapa I e Etapa II do experimento

\subsection{Análises laboratoriais e teste estatístico}

Os métodos analíticos utilizados foram descritos no Standard Methods for Examination of Water and Wastewater, $22^{\text {th }}$ Edition (APHA, 2012). As concentrações de amônia, nitrito, nitrato e fósforo, foram quantificados em cromatógrafo de íons (Dionex-100, coluna ASCR2_mm e CSCR2_mm). As determinações laboratoriais foram realizadas em triplicata no Laboratório de Saneamento da Escola Politécnica da USP. Foram realizadas análises de estatística descritiva básica, como desvio padrão, média e coeficiente de variação por meio do software MSExcel ${ }^{\circledR}$, (2013). Os resultados são apresentados em forma de tabelas e gráficos para melhor compreensão.

\section{RESULTADOS E DISCUSSÕES}

\subsection{Aclimatação do Lodo}

No início do sistema, o tanque de aeração foi preenchido com lodo biológico de um sistema de lodo ativado convencional (MBBR/IFAS) da EPUSP, tratando esgoto sanitário. A concentração inicial de oxigênio dissolvido foi mantida na faixa de 1,5-2,0 $\mathrm{mgO}_{2} / \mathrm{L}$, com troca volumétrica de $50 \%$, em ciclos iniciais de 6 horas. Após 20 dias de operação observou-se que o sistema estava em condições estáveis, com taxa de remoção de DQO e de nitrogênio superiores a $80 \%$, tendo sido considerado o término da etapa de aclimatação.

\subsection{Comportamento do processo de tratamento}

\subsubsection{Remoção de Material Orgânico}

A Figura 3 mostra a série temporal das análises de DQO durante as Etapas I e II do estudo. Os valores apresentados correspondem as médias das triplicatas determinadas durante o período amostral de 60 dias para cada etapa. 

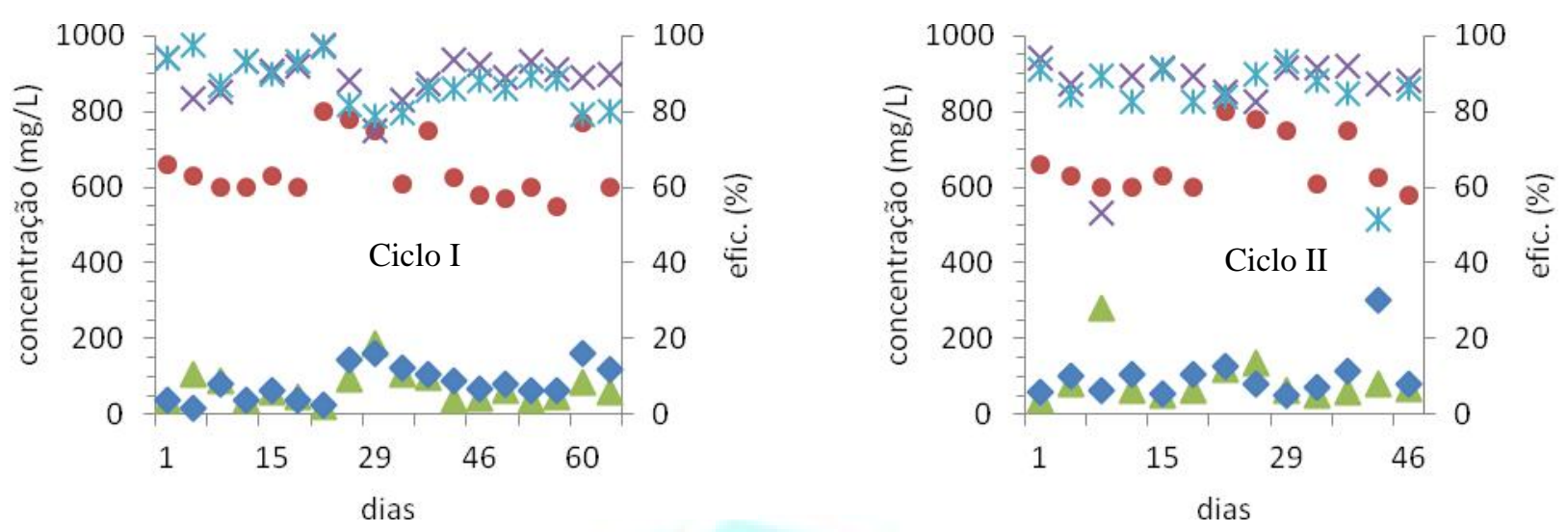

- Esgoto Bruto $\triangle$ SMBBR $\quad$ SBR $\quad \times \%$ Efic.SMBBR $\quad \% \%$ Efic. SBR

Figura 3. Série temporal - Análises de DQO total durante as Etapas I e II. Onde: • Concentração no esgoto bruto afluente; $\Delta$ Concentração no esgoto tratado SMBBR; - Concentração no esgoto tratado SBR; $\times$ Eficiência de remoção no sistema SMBBR; * Eficiência de remoção no sistema SBR.

Comparando-se os resultados de DQO afluente e efluente entre as etapas, pode ser verificado que tanto o SBR como o SMBBR foram mantidos sob operação estável e eficiente, mantendo ao longo do estudo uma eficiência superior a $87 \%$ SBR e $89 \%$ SMBBR na Etapa I e de 85\% SBR e $87 \%$ SMBBR na Etapa II onde foi operado com baixa concentração de OD. Estes resultados mostraram ainda, que a diminuição da concentração de OD não teve impacto negativo na eficiência de remoção de DQO e que o reator onde foi adicionado o material suporte (SMBBR) teve melhor desempenho em relação ao SBR. Resultados similares foram obtidos em reatores de MBBR com o material suporte similar ao utilizado neste estudo, como mostram os estudos realizados por Fujii (2011) e Fujii et al. (2013), que obtiveram eficiências superiores à 80\% de remoção de DQO.

Outros resultados similares foram encontrados por Nasser et al. (2012) operando sistema multifásico do tipo anaeróbio-anóxico-aeróbio $\left(\mathrm{A}^{2} / \mathrm{O}\right)$, no qual se mostrou que a variação da concentração de oxigênio dissolvido dentro da faixa de 0,5 a $2,5 \mathrm{mgO}_{2} / \mathrm{L}$ não apresenta efeito significativo na eficiência de remoção de matéria orgânica, mantendo-se na faixa de 85 a $87 \%$ em termos de DQO, o que confirma os resultados obtidos neste estudo.

A Figura 4 mostra o gráfico Box Whiskers das análises de DQO durante as Etapas I e II do estudo. Os valores apresentados correspondem as médias das triplicatas determinadas durante o período amostral e pode ser observado a dispersão dos dados assim como a concentração no efluente. De acordo com o representado na Figura 4, verifica-se que o efluente com baixa concentração de material orgânico, os valores de $\mathrm{DQO}$ foram de $82 \pm 45 \mathrm{mgO}_{2} / \mathrm{L}$ (SBR) e $70 \pm 39$ $\mathrm{mgO}_{2} / \mathrm{L}$ (SMBBR) na Etapa I e de $101 \pm 65 \mathrm{mgO}_{2} / \mathrm{L}(\mathrm{SBR})$ e $89 \pm 63 \mathrm{mgO}_{2} / \mathrm{L}(\mathrm{SMBBR})$ na Etapa II. 

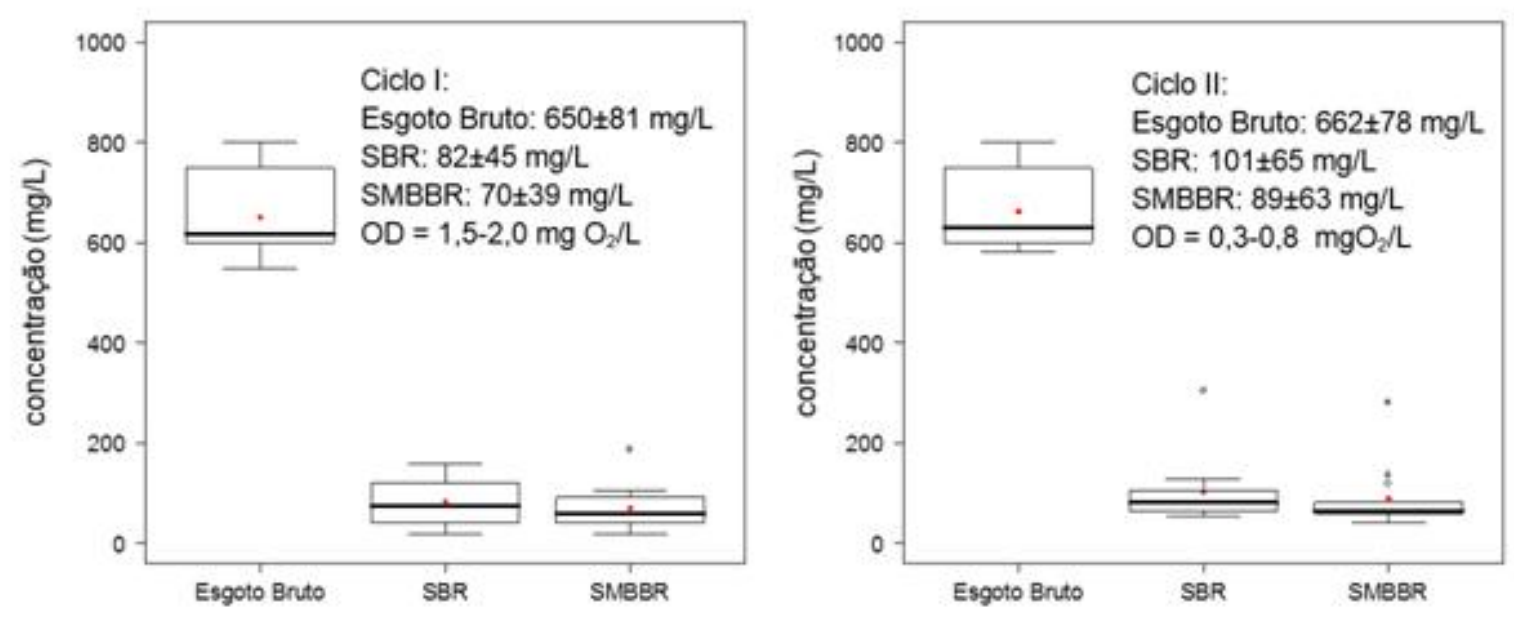

Figura 4. Box Whiskers - Análises de DQO total durante as Etapas I e II

Os resultados obtidos na Etapa II mostram mais uma vez que a baixa concentração de OD não afetou o desempenho do sistema. Observa-se, no entanto, ligeira elevação na concentração de DQO do efluente na Etapa II quando comparado com a Etapa I, esse fato pode ser relacionado com o aumento da concentração de DQO durante o estudo que passou de $650 \mathrm{mgO}_{2} / \mathrm{L}$ na Etapa I para $662 \mathrm{mgO}_{2} / \mathrm{L}$ na Etapa II.

A Figura 5 mostra a série temporal das análises de DQO solúvel durante as Etapas I e II do estudo. Os valores apresentados correspondem as médias das triplicatas determinadas durante o período amostral de 60 dias para cada etapa.
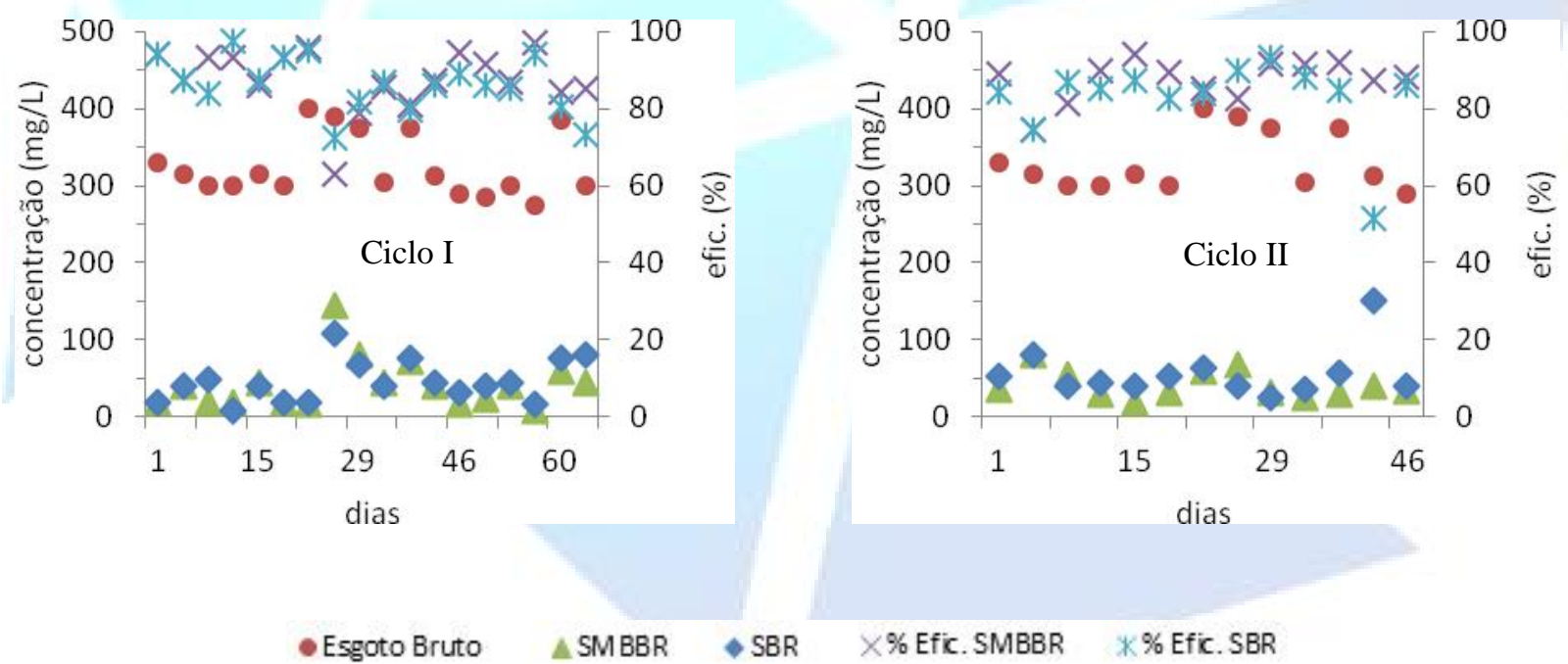

$\times \%$ Efic. SMBBR $\quad * \%$ Efic. SBR

Figura 5. Série temporal - Análises de DQO solúvel durante as Etapas I e II. Onde: • Concentração no esgoto bruto afluente; $\triangle$ Concentração no esgoto tratado SMBBR; $\bullet$ Concentração no esgoto tratado SBR; $\times$ Eficiência de remoção no sistema SMBBR; * Eficiência de remoção no sistema SBR. 
Comparando-se os resultados de DQO solúvel afluente e efluente entre as etapas, pode ser dito que tanto o SBR como o SMBBR tiveram boa remoção de compostos orgânicos solúveis de fácil degradação, correspondendo a parcela de material orgânico no esgoto que é facilmente degradada. Os dados da série histórica da Figura 5 demonstram uma estabilidade operacional do sistema que manteve eficiência média de remoção de DQO superior a $90 \%$ em ambos os ensaios. A Figura 6 mostra os dados em forma de gráfico Box Whiskers das análises de DQO durante as Etapas I e II do estudo.
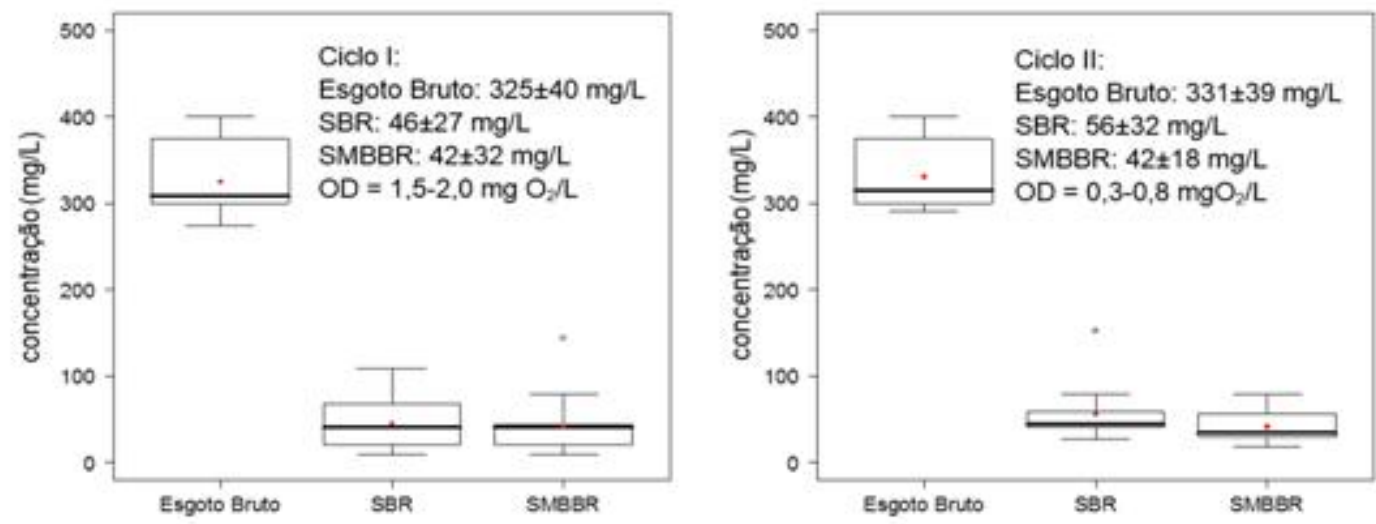

Figura 6. Box e Whiskers - Análises de DQO solúvel durante as Etapas I e II

Os valores apresentados correspondem as médias das triplicatas determinadas durante o período amostral e pode ser observado a dispersão dos dados assim como a concentração no efluente. Analisando as concentrações de DQO solúvel no efluente, verifica-se que os valores médios inferiores a $56 \mathrm{mgO}_{2} / \mathrm{L}$ correspondem a compostos de difícil degradação, ou seja, corresponde a fração de material dissolvido não biodegradável presente no esgoto. A diminuição do oxigênio e das condições operacionais adotada não teve nenhum tipo de influência nessa questão, sendo o comportamento dos sistemas similares em ambos os ensaios.

\subsubsection{Oxidação de compostos nitrogenados}

A Figura 7 mostra a série temporal das concentrações de NTK obtidas nas Etapas I e II do experimento. Os dados representam as médias das análises em triplicatas realizadas durante o período do estudo.
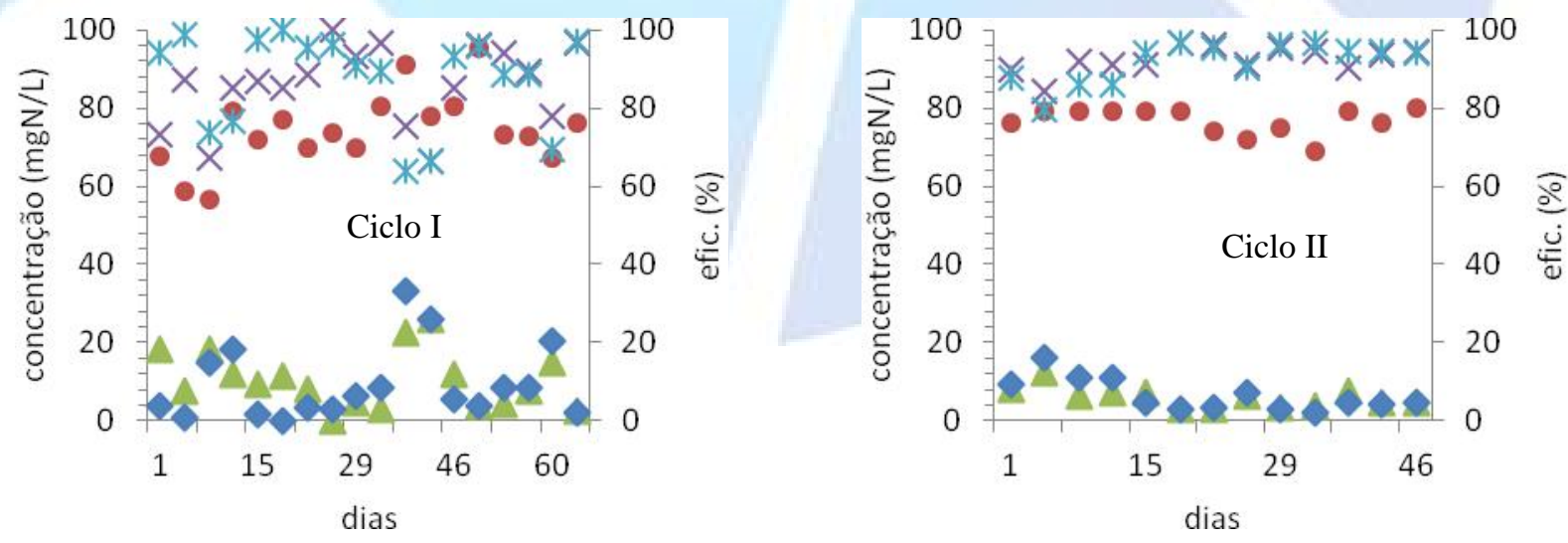

- Esgoto Bruto $\quad \triangle$ SMBBR $\quad$ SBR $\quad \times \%$ Efic.SMBBR $\quad \%$ Efic. SBR

Figura 7. Série temporal - Análises de NTK durante as Etapas I e II. Onde: • Concentração no esgoto bruto afluente; $\Delta$ Concentração no esgoto tratado SMBBR; $\bullet$ Concentração no esgoto tratado SBR; $\times$ Eficiência de remoção no sistema SMBBR; * Eficiência de remoção no sistema SBR. 
Ao analisar os dados de NKT obtidos nos sistemas de SBR e SMBBR na Etapa I e na Etapa II, verifica-se que houve oxidação do nitrogênio amoniacal e orgânico ao longo de todo o estudo. No sistema de SMBBR, as concentrações de NKT no afluente resultou em valores inferiores à $20 \mathrm{mgN} / \mathrm{L}$ em todo o período, evidenciando a estabilidade operacional do sistema e ainda, que a redução da concentração de $\mathrm{OD}$ de $1,5-2,0$ para $0,3-0,8 \mathrm{mgO}_{2} / \mathrm{L}$ não afeta o processo de nitrificação.

A demanda de oxigênio disponível foi suficiente para os organismos nitrificantes realizarem a oxidação dos compostos nitrogenados, sem que haja limitação no processo. Resultados similares foram obtidos nos estudos de Bueno (2011) onde houve remoção de nitrogênio superior a 90\% e um afluente com concentrações inferiores a $10 \mathrm{mg} / \mathrm{L}$, sob aeração de 0,3 a $0,5 \mathrm{mgO}_{2} / \mathrm{L}$. Nos estudos de Guo et al. (2011), que operou um sistema em bateladas sequenciais de $10 \mathrm{~L}$ alternando-se as etapas anaeróbias e aeróbias, esta última mantendo-se o OD na faixa de 0,1 a 0,6 mg/L, obteve $78 \%$ de remoção de nitrogênio. Ainda, o processo de SMBBR na Etapa II se mostrou bastante estável, com eficiências de remoção superiores à $90 \%$ de $\mathrm{NKT}_{\text {e }} \mathrm{NH}_{3}$, conferindo segurança e confiabilidade ao sistema de tratamento em termos de nitrificação, fato que pode ser observado na Figura 8.

A Figura 9 mostra a série temporal das concentrações de amônia $\left(\mathrm{N}_{-} \mathrm{NH}_{3}{ }^{-}\right)$e a Figura $10 \mathrm{o}$ gráfico Box Whiskers, sendo que os valores apresentados correspondem as médias das triplicatas determinadas durante o período amostral.
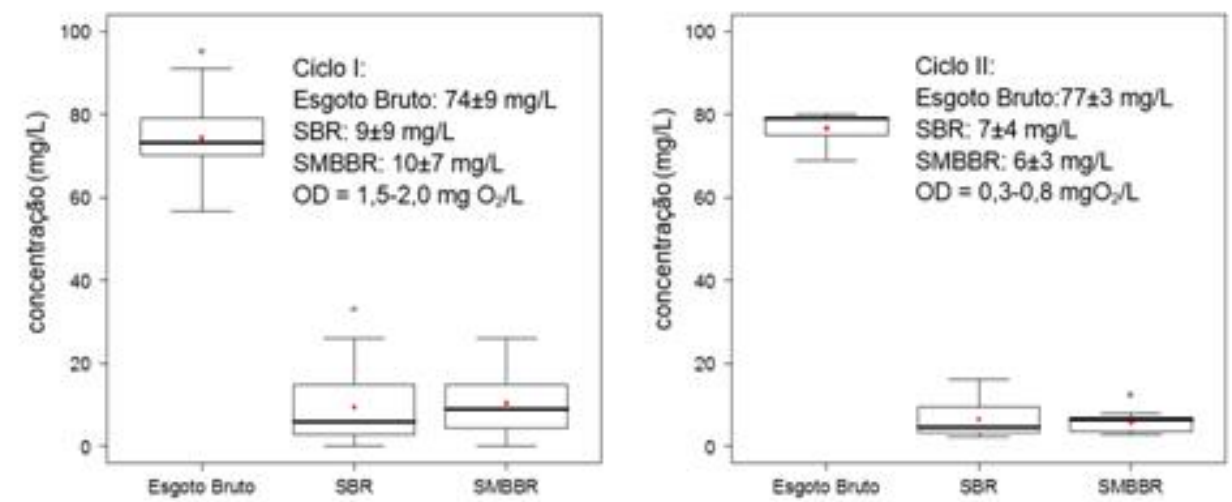

Figura 8. Box e Whiskers - Análises de NTK durante as Etapas I e II
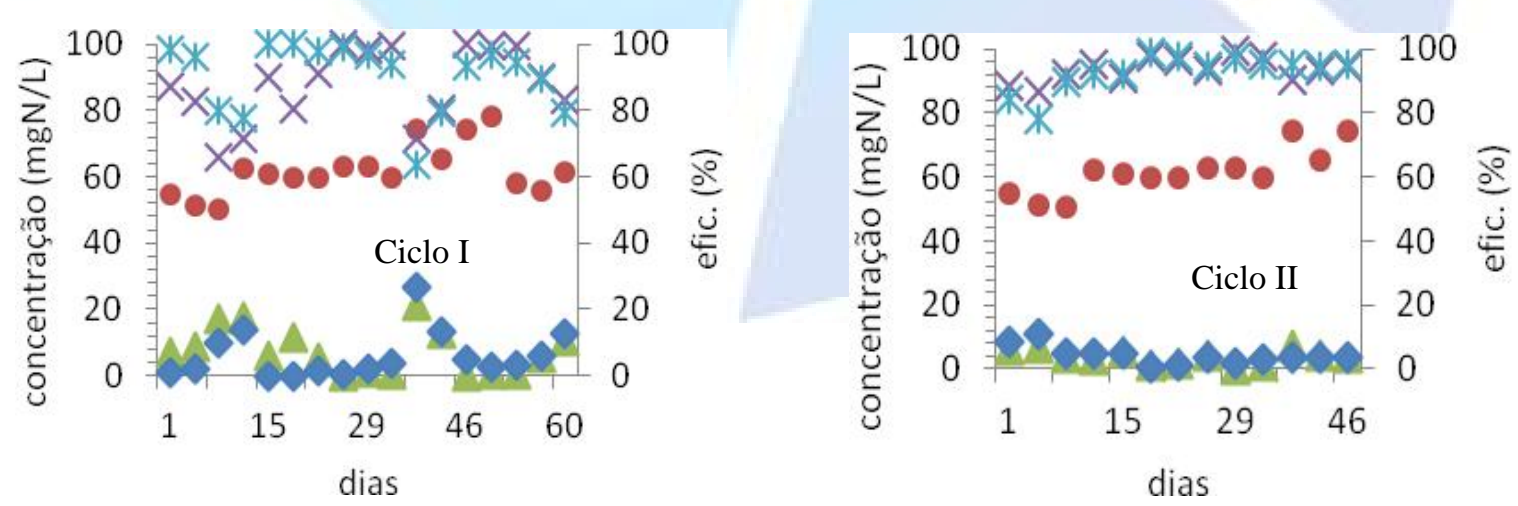

- Esgoto Bruto $\quad \triangle$ SMBBR $\quad$ SBR $\quad \times \%$ Efic.SMBBR $\quad$ \% Efic. SBR

Figura 9. Série temporal - Análises de $\mathrm{NH}_{3}{ }^{-}$durante as Etapas I e II. Onde: • Concentração no esgoto bruto afluente; $\Delta$ Concentração no esgoto tratado SMBBR; $\bullet$ Concentração no esgoto tratado SBR; $\times$ Eficiência de remoção no sistema SMBBR; * Eficiência de remoção no sistema SBR. 

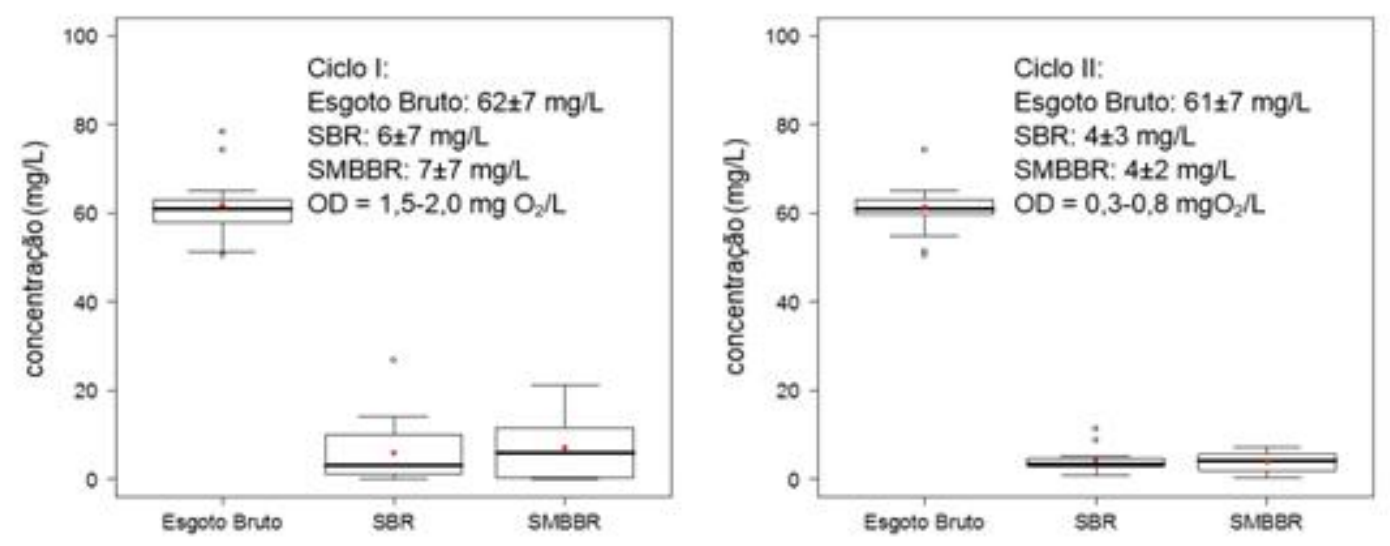

Figura 10. Box e Whiskers - Análises de $\mathrm{NH}_{3}{ }^{-}$durante as Etapas I e II

A elevada capacidade de nitrificação obtida nos processos de SBR e SMBBR nas Etapas I e II podem ser confirmadas na série temporal de concentração de nitrogênio amoniacal presente no efluente (Figura 9). A Figura 10 mostra os dados em forma de gráfico Box Whiskers das análises de nitrogênio amoniacal durante as Etapas I e II do estudo.

Quando se comparam os resultados médios de concentração de nitrogênio amoniacal (Figura 10), fica evidente a grande capacidade de nitrificação e estabilidade operacional do processo de SMBBR operado na Etapa II. Esta capacidade pode ser justificada pela presença da biomassa aderida, que além de garantir uma concentração de biomassa maior do que a obtida no reator SBR (apenas com biomassa em suspensão), possui melhor estabilidade operacional e idade do lodo elevada, favorecendo o desenvolvimento das bactérias nitrificantes. As concentrações de nitrito $\left(\mathrm{NO}_{2}{ }^{-}\right)$no esgoto sanitário e no efluente tratado foram menores que $0,5 \mathrm{mgN} / \mathrm{L}$ que, para todos os efeitos, foram desconsideradas. A Figura 11 mostra a média das concentrações de nitrato $\left(\mathrm{NO}_{3}{ }^{-}\right)$ obtidas nas Etapas I e II do experimento.

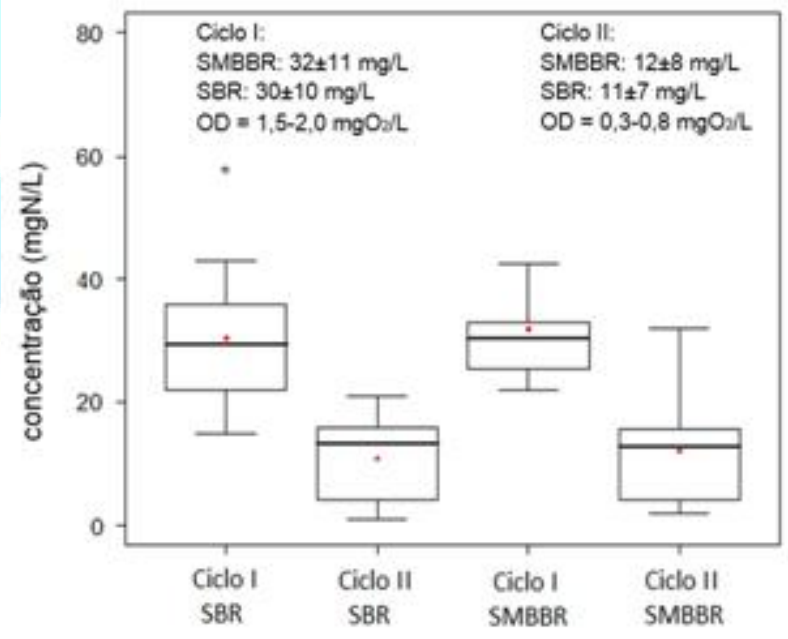

Figura 11. Box e Whiskers - Análises de Nitrato durante as Etapas I e II

As médias das concentrações de $\mathrm{NO}_{3}{ }^{-}$foram consideravelmente baixas na Etapa II (menores que $12 \mathrm{mgN} / \mathrm{L}$ ), o que não ocorreu na Etapa I, com valores médios menores que $32 \mathrm{mgN} / \mathrm{L}$ em ambos os processos. Essa alta capacidade de desnitrificação obtida na Etapa II em ambos os processos de SBR e SMBBR pode ser justificada pela estratégia de operação estabelecida no ciclo II. Nesse caso, a baixa concentração de OD $\left(0,3-0,8 \mathrm{mgO}_{2} / \mathrm{L}\right)$ na etapa de reação (aeróbia/anóxica) potencializou o processo de desnitrificação durante o experimento. 
Resultados semelhantes foram obtidos nos estudos realizados por Munch et al. (1996) e Guo et al. (2007) com OD na faixa de 0,5 mg/L e tempo de reação aerado/anóxico de $170 \mathrm{~min}$. No estudo de Nasser et al. (2012), o aumento da concentração de OD no licor misto de $0,5 \mathrm{mgO} / \mathrm{L}$ para a faixa de 1,5 a 2,5 $\mathrm{mgO}_{2} / \mathrm{L}$ acarretou a elevação da taxa de acumulação de $\mathrm{NO}_{\mathrm{x}}-\mathrm{N}$, indicando inibição do processo de desnitrificação. A taxa combinada de nitrificação e desnitrificação obtida neste estudo foi de $71 \%$ para $0,5 \mathrm{mgO}_{2} / \mathrm{L}$ e de apenas $40 \%$ para $2,0 \mathrm{mgO}_{2} / \mathrm{L}$. Considerando-se que a taxa de nitrificação foi semelhante para os dois níveis de oxigênio dissolvido (ligeiramente superior para 2,0 $\mathrm{mgO}_{2} / \mathrm{L}$ ), essa diferença decorre principalmente da menor taxa de desnitrificação apresentada quando há mais oxigênio dissolvido no licor misto. A Figura 12 mostra a média das concentrações de alcalinidade obtidas nas Etapas I e II do experimento.

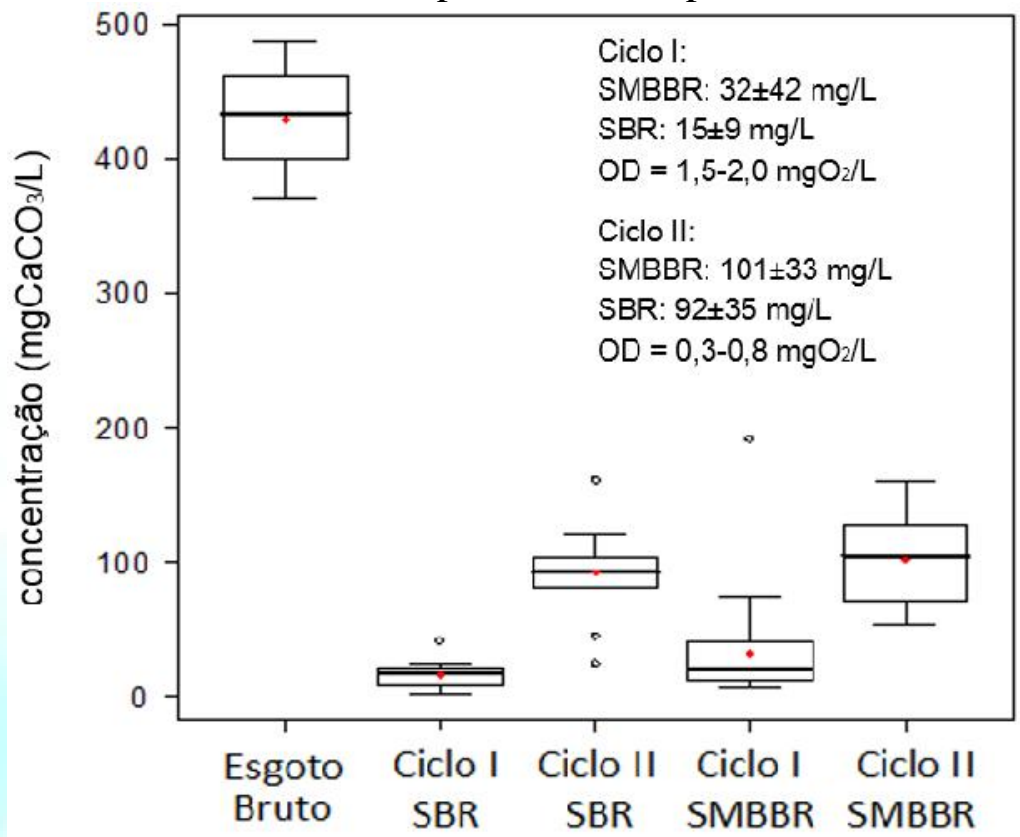

Figura 12. Box e Whiskers - Análises de Alcalinidade durante as Etapas I e II

A concentração média no efluente final nos sistemas SBR e SMBBR na Etapa I foi de $15 \pm 9$ e $32 \pm 42 \mathrm{mgCaCO}_{3} / \mathrm{L}$ e na Etapa II foi de $92 \pm 35$ e $101 \pm 33 \mathrm{mgCaCO}_{3} / \mathrm{L}$, respectivamente. Esses dados demonstram que na Etapa II, houve reposição da alcalinidade pelo processo de desnitrificação. Resultados semelhantes foram obtidos no estudo realizado por Li et al. (2007) em um sistema SBR operado com diferentes concentrações de OD: quando operado com OD inferior a $1,0 \mathrm{mg} / \mathrm{L}$ obteve uma boa recuperação de alcalinidade sem comprometer o processo de nitrificação. Os processos de transformação das formas nitrogenadas (amonificação, nitrificação e desnitrificação) são os mais influentes no sistema quanto à variação da alcalinidade, e consequentemente na manutenção do $\mathrm{pH}$ do licor misto. Assim, é possível fazer um balanço de massa da alcalinidade no sistema a partir da avaliação destes processos. Para tanto, considera-se que $1,0 \mathrm{mgNH}_{4}{ }^{-}$demanda $7,14 \mathrm{mg} / \mathrm{L}$ de alcalinidade em termos de $\mathrm{CaCO}_{3}$ para o processo de nitrificação (há produção de $\mathrm{H}^{+}$), ao passo que os processos de amonificação e desnitrificação fornecem 3,57 $\mathrm{mg} \mathrm{CaCO}_{3} / \mathrm{L}$ (neles há consumo de $\mathrm{H}^{+}$). Quando as variações de nitrito são negligenciadas, é possível observar que o balanço de alcalinidade no sistema é compatível, descartando a necessidade da introdução de alcalinizante artificial - o que se fez necessário na Etapa I.

\subsubsection{Remoção de Fósforo}

A Figura 13 mostra a série temporal das concentrações de fósforo total obtidas no experimento. Os valores correspondem a média das triplicatas realizadas durante o período do estudo. 

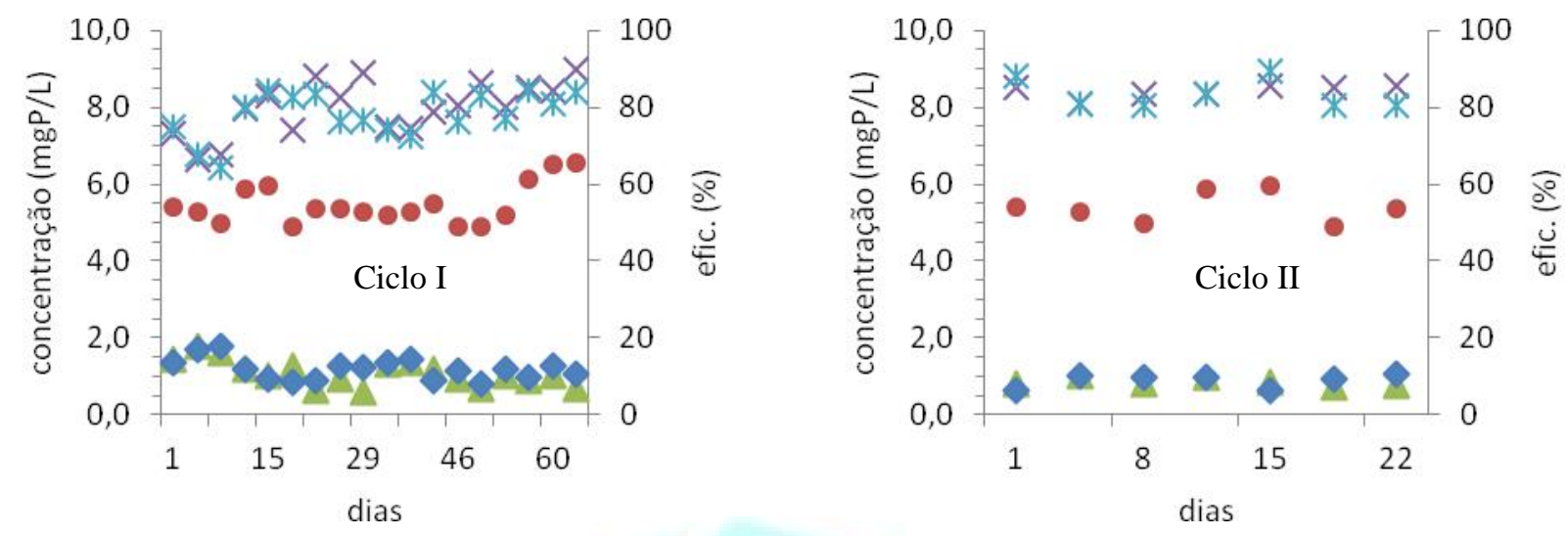

- Esgoto Bruto $\quad \triangle$ SMBBR $\quad$ SBR $\quad \times \%$ Efic.SMBBR $\quad \% \%$ Efic.SBR

Figura 13. Série temporal - Análises de Fósforo total durante as Etapas I e II. Onde: • Concentração no esgoto bruto afluente; $\Delta$ Concentração no esgoto tratado SMBBR; $\bullet$ Concentração no esgoto tratado SBR; $\times$ Eficiência de remoção no sistema SMBBR; * Eficiência de remoção no sistema SBR.

Verificando-se os resultados obtidos, Figura 13, tanto o SBR como o SMBBR apresentaram efluente com baixas concentrações deste elemento durante todo o período de estudo. A concentração média no efluente final nos sistemas SBR e SMBBR na Etapa I foi de 1,2 $\pm 0,3$ e $1,1 \pm 0,3 \mathrm{mgP} / \mathrm{L}$ e na Etapa II foi de $0,9 \pm 0,2$ e $0,9 \pm 0,1 \mathrm{mgP} / \mathrm{L}$, respectivamente. A eficiência de remoção de fósforo foi, portanto, de $78 \%$ para o sistema SBR e de $80 \%$ para o SMBBR com OD entre 1,5 e 2,0 mg/L (Etapa I) e de 83\% para ambos os sistemas na Etapa II, com OD entre 0,3 e 0,8 $\mathrm{mg} / \mathrm{L}$.

A pesquisa de Guo et al. (2011) demonstrou que a eficiência de remoção de fósforo está ligada à relação estequiométrica entre o carbono e os nutrientes $(\mathrm{N}$ e $\mathrm{P})$ presentes no esgoto sanitário afluente ao tratamento. Isso ocorre porque os processos de remoção de fósforo (especificamente a etapa de liberação de fósforo no ambiente anaeróbio) e de remoção de nitrogênio (etapa de desnitrificação) competem por carbono. O sistema em bateladas sequenciais alternando condições anaeróbias e aeróbias foi operado com concentração de OD entre 0,1 e 0,6 mg/L na etapa aeróbia. A eficiência de remoção de fósforo subiu de $40 \%$ (C/N de 1,5; C/P de 8,9 e fosfato efluente de $9 \mathrm{mg} / \mathrm{L})$ para $73 \%(\mathrm{C} / \mathrm{N}$ de 2,6; C/P de 15 e fosfato efluente de $4 \mathrm{mg} / \mathrm{L})$ e $95 \%$ (C/N de 4,1; C/P de 24 e fosfato efluente de $1 \mathrm{mg} / \mathrm{L}$ ).

\section{CONCLUSÕES}

Dentre os aspectos mais importantes que foram observados, destaca-se o desempenho alcançado pela tecnologia SMBBR quando o sistema foi operado em baixas concentrações de OD, em termos de eficiências na remoção de nitrogênio e fósforo, bem como sua maior estabilidade operacional em comparação com o sistema de SBR. Os resultados demonstram que é possível operar o sistema com baixo oxigênio dissolvido, potencializando o processo de desnitrificação sem trazer prejuízos na remoção de matéria orgânica e na nitrificação, resultando em um ganho econômico relativo à redução de energia elétrica para aeração. Na etapa anaeróbia, essa metodologia pode ser feita para avaliar também a atuação dos OAPs desnitrificantes (OAPDs), cuja existência e atuação vem sendo estudada mais recentemente por diversos autores. 


\section{REFERENCIAS}

ACCINELLI, C.; SACCÀ, M. L.; MENCARELLI, M.; VICARI, A. Application of bioplastic moving bed biofilm carriers for the removal of synthetic pollutants from wastewater. Bioresource technology. New York, v.120, p:180-186, 2012.

ANDREOTTOLA, G.; FOLADORI, P.; RAGAZZI, M. Upgrading of a small wastewater treatment plant in a cold climate region using a moving bed biofilm reactor (MBBR) system. Water science and technology. EUA, v. 41, n. 1, p. 177-185, 2000.

APHA, AWWA, WEF. Standard Methods for examination of water and wastewater. $22^{\text {nd }}$. Washington: American Public Health Association. EUA, 2012, 1360 pp. ISBN 978-087553013-0.

AYGUN, A.; NAS, B.; BERKTAY, A. Influence of high organic loading rates on COD removal and sludge production in moving bed biofilm reactor. Environmental Engineering Science. New York, v.25, n.9, p.1311-1316, 2008.

BUENO, R. F. Nitrificação e desnitrificação simultânea em reator com biomassa em suspensão e fluxo contínuo de esgoto. 2011. Dissertação (Mestrado em Saúde Ambiental) - Faculdade de Saúde Pública, Universidade de São Paulo, São Paulo, 2011. Disponível em: <http://www.teses.usp.br/teses/disponiveis/6/6134/tde-09092011-153620/>. Acesso em: 2015-1004.

CHIU, Y.C.; LEE, L.L.; CHANG, C.N.; CHAO, A.C. Control of carbon and ammonium ratio for simultaneous nitrification and denitrification in a sequencing batch bioreactor. International biodeterioration \& biodegradation. New York, v.59, n.1, p.1-7, 2007.

FUJII, F.Y. Análise comparativa entre o processo de lodo ativado e o reator de biofilme de leite móvel na remoção de nitrogênio de esgoto sanitário. 2011. Dissertação (Mestrado em Engenharia Hidráulica) - Escola Politécnica, Universidade de São Paulo, São Paulo, 2011. Disponível em: <http://www.teses.usp.br/teses/disponiveis/3/3147/tde-12122011-134438/>. Acesso em: 2015-1004 .

FUJII, F.Y.; PIVELI, R.P.; CAMPOS, F.; BUENO, R.F.; SOBRINHO, P.A. Desempenho de reator integrado de lodo ativado com biofilme em leito móvel. Revista de Engenharia Sanitária e Ambiental - (RESA). São Paulo, v.18, n.4, p.349-358, 2013.

GIESEKE, A.; ARNZ, P.; AMANN, R.; SCHRAMM, A. Simultaneous P and N removal in a sequencing batch biofilm reactor: insights from reactor-and microscale investigations. Water Research. EUA, v.36, n.2, p.501-509, 2002.

GUO, H.; ZHOU, J.; ZHANG, S.; GUO, Z. Characteristics of Nitrogen and Phosphorus removal in a sequencing batch reactor. Journal of Environmental Science. EUA, v.23, p.110-113, 2011.

HELNESS, H.; ØDEGAARD, H. Biological phosphorus and nitrogen removal in a sequencing batch moving bed biofilm reactor. Water Science and Technology. EUA, v.43, n.1, p.233-240, 2001. 
HEM L.J.; RUSTEN, B.; ØDEGAARD, H. Nitrification in a moving bed biofilm reactor. Water Research. EUA, v.28, n.6, p.1425-1433, 1994.

GUO, J.; YANG, Q.; PENG, Y.; YANG, A.; WANG, S. Biological nitrogen removal with real-time control using step-feed SBR technology. Enzyme and Microbial Technology. EUA, v.40, p.15641569, 2007.

KIM, B.K.; CHANG, D.; SON, D.J.; KIM, D.W.; CHOI, J.K.; YEON, H.J.; YOON, C.Y.; FAN, Y.; LIM, S.Y.; HONG, K.H. Wastewater treatment in moving bed biofilm reactor operated by flow reversal intermittent aeration system. World Academy Science, Engineering and Technology. EUA, v.60: p.581-584, 2011.

LI, B.; IRVIN, S. The comparison of alkalinity and ORP as indicators for nitrification and denitrification in a sequencing batch reactor (SBR). Biochemical Engineering Journal. EUA, v.34, n.3, p.248-255, 2007.

MUNCH, E.V.; LANT, P.; KELLER, J. Simultaneous nitrification and denitrification in benchscale sequencing bath reactors. Water Research. EUA, v.30, n.2, p.277-284, 1996.

NASEER, R.; ABUALHAIL, S.; XIWU, L. Biological nutrient removal with limited organic matter using a novel anaerobic-anoxic/oxic multi-phased activated sludge process. Saudi Journal of Biological Sciences. EUA, v.20, n.1, p.11-21, 2013.

ØDEGAARD, H.; RUSTEN, B. WESTRUM, T. A new moving bed biofilm reactor-applications and results. IAWQ Second International Conference on Biofilm Reactors. Paris, 27 September to 29 October, v.1, pp.221-229, 1993.

RUSTEN, B.; HEM, L.J.; ØDEGAARD, H. Nitrification of municipal wastewater in moving-bed biofilm reactors. Water Environment Research. EUA, v.67, n.1, p.75-86, 1995.

THAURÉ, D.; LEMOINE, C.; DANIEL, O.; MOATAMRI, N.; CHABROL, J. Optimisation of aeration for activated sludge treatment with simultaneous nitrification denitrification. Water Science Technology. EUA, v.58, n.3, p.639-645, 2008.

WENTZEL, M.C.; EKAMA, G.A.; DOLD, P.L.; MARAIS, G.V.R. Biological phosphorus removal - Steady state process design. Journal Water SA. EUA, v.16, n.1, p.29-48, 1990.

ZENG, R.J.; LEMAIRE, R.; YUAN, Z.; KELLER, J. Simultaneous nitrification, denitrification, and phosphorus removal in a lab-scale sequencing batch reactor. Biotechnology and bioengineering. EUA, v.84, n.2, p.170-178, 2003.

ZHANG, P.; QI, Z.H.O.U. Simultaneous nitrification and denitrification in activated sludge system under low oxygen concentration. Frontiers of environmental science \& engineering in China. China, v.1, n.1, p.49-52, 2007.

ZHAO, H.W.; MAVINIC, D.S.; OLDHAM, W.K.; KOCH, F.A.. Controlling factors for simultaneous nitrification and denitrification in a two-stage intermittent aeration process treating domestic sewage. Water Research. EUA, v.33, n.4, p.961-970, 1999. 